\section{Mortalidade infantil segundo cor ou raça com base no Censo Demográfico de 2010 e nos sistemas nacionais de informação em saúde no Brasil}

\section{Infant mortality according to color or race based on the 2010 Population Census and national health information systems in Brazil}

\section{Mortalidad infantil según color de piel o raza, en base al Censo Demográfico de 2010 y en los sistemas nacionales de información en salud de Brasil}

\author{
Aline Diniz Rodrigues Caldas 1 \\ Ricardo Ventura Santos 2 \\ Gabriel Mendes Borges 3 \\ Joaquim Gonçalves Valente 2 \\ Margareth Crisóstomo Portela 2 \\ Gerson Luiz Marinho 4
}

doi: 10.1590/0102-311X00046516

\section{Resumo}

O objetivo deste estudo é investigar as informações sobre mortalidade infantil no Brasil, de acordo com a cor ou raça com foco nos indígenas, baseando-se nos dados do Censo Demográfico de 2010 e daqueles oriundos do Sistema de Informação sobre Mortalidade (SIM) e do Sistema de Informações sobre Nascidos Vivos (SINASC). Em ambas as fontes, as taxas de mortalidade infantil (TMI) de indígenas foram as mais elevadas dentre os diversos segmentos populacionais. Apesar dos dados censitários indicarem desigualdades de cor ou raça, as TMI para indígenas e pretos foram inferiores às derivadas do SIM/SINASC. Particularidades metodológicas quanto à coleta dos dados em ambas as fontes devem ser consideradas. A redução da TMI no Brasil nas últimas décadas é em larga medida atribuída à prioridade da saúde infantil na agenda politica. Os achados deste trabalho são indicativos de que o impacto das políticas públicas não alcançou os indígenas em mesma escala que o restante da população. Novas fontes de dados relativos à ocorrência de óbitos nos domicílios, de abrangência nacional, como é o caso do Censo de 2010, podem contribuir para uma melhor compreensão das desigualdades segundo cor ou raça no Brasil.

Índios Sul-americanos; Saúde de Populações Indígenas; Mortalidade Infantil; Desigualdades em Saúde

\author{
Correspondência \\ A. D. R. Caldas \\ Rua Mariz e Barros 272, apto. 501, Niterói, RJ 24220-121, \\ Brasil. \\ alinediniz.caldas@gmail.com \\ 1 Núcleo Estadual do Ministério da Saúde no Rio de Janeiro, \\ Ministério da Saúde, Rio de Janeiro, Brasil. \\ 2 Escola Nacional de Saúde Pública Sergio Arouca, Fundação \\ Oswaldo Cruz, Rio de Janeiro, Brasil. \\ 3 Instituto Brasileiro de Geografia e Estatística, \\ Rio de Janeiro, Brasil. \\ 4 Escola de Enfermagem Anna Nery, Universidade Federal do \\ Rio de Janeiro, Rio de Janeiro, Brasil.
}




\section{Introdução}

A taxa de mortalidade infantil (TMI) é amplamente reconhecida como um importante indicador das condições de vida de uma população ${ }^{1}$. No Brasil, aconteceu uma substantiva redução da mortalidade infantil ao longo das últimas décadas, passando de 47,1 para 14,6 óbitos/1.000 nascidos vivos entre 1990 e 2012 2, com decréscimos em todas as regiões do país. Nesse período, as desigualdades das TMI regionais também diminuíram, assim como, em menor grau, as disparidades sociais 3 .

Nas investigações epidemiológicas, a TMI é comumente analisada segundo componentes, quais sejam: neonatal precoce (óbitos ocorridos entre 0 e 6 dias de vida, por mil nascidos vivos); neonatal tardio (entre 7 e 27 dias) e pós-neonatal (entre 28 e 364 dias) 4 . A redução da mortalidade infantil no Brasil vem ocorrendo principalmente no componente pós-neonatal 4. Dentre os fatores envolvidos, destacam-se melhorias nas condições socioeconômicas e na oferta de serviços de saúde, assim como mudanças demográficas, como a redução da fecundidade. Por sua vez, as taxas de mortalidade no período neonatal apresentaram tendência de declínio mais lento 5,6.

Há relativamente poucos estudos voltados para análises comparativas acerca da mortalidade infantil segundo recorte étnico-racial no Brasil 7,8,9,10. Entretanto, em períodos recentes, no plano dos debates sobre determinantes sociais, essa questão tem merecido crescente destaque. De uma maneira geral, as análises apontam para valores de TMI mais elevados no caso das crianças classificadas como pretas, pardas e indígenas, mesmo após ajustes por covariáveis como baixo peso ao nascer e as relativas à situação socioeconômica 8,11,12,13

No que diz respeito à população indígena do país, a inclusão da categoria “indígena" no quesito cor ou raça dos censos decenais e de outras pesquisas de representatividade nacional realizadas pelo Instituto Brasileiro de Geografia e Estatística (IBGE), a partir de 1991, tem possibilitado uma importante ampliação das fontes de dados sobre este segmento populacional 14,15,16,17. Vale destacar que, no Censo Demográfico de 2010, a pergunta sobre cor ou raça foi também incluída no questionário básico, permitindo que toda a população fosse classificada segundo tal variável 18. Esse ponto é particularmente relevante para os indígenas, pois é o segmento de cor ou raça de menor tamanho, não alcançando 1 milhão de pessoas (<0,5\% da população brasileira total). Desse modo, a geração de informações com base nos dados do universo do Censo potencialmente contribui para um melhor conhecimento das características populacionais.

Com relação à produção de dados sobre mortalidade nos levantamentos censitários, outro destaque é que no Censo de 2010 foram incluídas questões relacionadas à ocorrência de óbito nos domicílios particulares no ano anterior à entrevista censitária 19. Ademais, tal como nos censos anteriores, foram mantidos os quesitos sobre filhos nascidos vivos e sobreviventes, o que permite o cálculo da mortalidade infantil usando-se técnicas demográficas indiretas 19.

Com foco nos indígenas, o presente trabalho tem por objetivo estimar e comparar as TMI segundo cor ou raça no Brasil, baseando-se em dados do Censo de 2010, do SIM (Sistema de Informação sobre Mortalidade) e do SINASC (Sistema de Informações sobre Nascidos Vivos), em 2009-2010. Esse período foi escolhido com vistas a garantir a sobreposição temporal dos dados das diferentes fontes utilizadas. Além disso, são apresentadas análises acerca dos componentes da mortalidade infantil segundo cor ou raça computadas com base nos dados do SIM e do SINASC.

\section{Métodos}

O Censo de 2010 envolveu a coleta de dados em 67,6 milhões de domicílios nos 5.565 municípios do país, tendo como referência a data de 31 de julho de 2010. As entrevistas foram realizadas utilizandose dois tipos de questionário, denominados de "básico" e "da amostra". O questionário básico apresentava 37 quesitos e foi aplicado em todos os domicílios ocupados não selecionados para a aplicação do questionário da amostra, que incluía 108 quesitos. Todas as perguntas do questionário básico também estavam presentes no questionário da amostra, de forma que se almejava aplicar o conjunto de 37 quesitos em todos os domicílios do país 18,19 .

O questionário básico contemplou perguntas sobre características demográficas e socioeconômicas dos moradores, assim como referentes aos domicílios. No caso dos indígenas, incluiu questões 
relativas à etnia e línguas faladas ${ }^{17}$. Neste trabalho, a classificação de indígena envolveu somente a pergunta sobre cor ou raça, não tendo sido incluídos aqueles indivíduos classificados como indígenas por meio do quesito "6.05 - você se considera indígena?". Essa pergunta foi feita para todos os indivíduos que, residentes em terras indígenas, se classificaram como branco, preto, amarelo ou pardo no quesito cor ou raça 17 . Portanto, para fins deste estudo, a população indígena incluiu tanto aquela residente nas terras indígenas quanto fora delas, nas áreas urbanas e rurais, ou seja, a totalidade dos que se autodeclararam indígenas segundo critério cor ou raça em todo o território nacional.

Quanto à mortalidade, o Censo de 2010 coletou dados acerca da ocorrência de óbitos nos domicílios, incluindo sexo e idade do falecido, nos 12 meses anteriores a 31 de julho de 2010 (ou seja, óbitos ocorridos de 1o de agosto de 2009 a 31 de julho de 2010) 19. Uma vez que não foram coletados dados acerca da cor ou raça do indivíduo falecido, atribuiu-se ao óbito a mesma cor ou raça do morador identificado como o responsável pelo domicílio.

Os dados de óbitos do Censo de 2010 se referem ao universo e foram obtidos por meio do Banco Multidimensional de Estatísticas (BME; http://www.bme.ibge.gov.br, acessado em 17/Abr/2013). Para o cálculo das TMI, foram selecionadas as frequências de óbitos de menores de um ano ocorridos nos domicílios particulares. Os óbitos foram corrigidos pelos fatores de ajuste propostos por Queiroz \& Sawyer 20. Para compor os denominadores das TMI, foram selecionadas as frequências da variável V6664 (filho(s) nascido(s) vivo(s) no período de 12 meses anteriores a 31 de julho de 2010), segundo a cor ou raça do responsável pelo domicílio. Tais frequências foram geradas valendo-se de microdados da amostra do Censo de 2010, disponibilizados segundo Unidade da Federação (UF) no site do IBGE, utilizando-se, para as estimativas, procedimentos para amostras complexas do programa SAS, versão 9.3 (SAS Inst., Cary, Estados Unidos).

Também baseando-se nos dados do Censo de 2010, foram estimadas as TMI segundo o método dos filhos sobreviventes. Tal procedimento é uma técnica demográfica indireta desenvolvida por Brass \& Coale 21, que utiliza informações coletadas nos censos e em pesquisas domiciliares para calcular a probabilidade de morte de crianças desde o nascimento até certa idade. Visa a corrigir problemas nos dados, como o sub-registro de óbitos infantis. Com base nas informações de filhos tidos nascidos vivos e filhos sobreviventes levantadas no Censo de 2010, é possível calcular a proporção de mortes de crianças nascidas de mães em cada grupo etário quinquenal. Utilizando-se a variante proposta por Coale \& Trussel 22, aloca-se temporalmente cada uma dessas estimativas. Partindo-se do pressuposto de que seguem uma variação monotônica no tempo, o método envolve ajustar uma regressão logística a fim de suavizar as estimativas de acordo com as informações da data a que cada uma se refere, além de desconsiderar os valores para os grupos etários abaixo de 25 anos. As TMI estimadas para cada grupo de cor ou raça foram ajustadas proporcionalmente de forma a reproduzir a TMI para o Brasil divulgada pelo IBGE 23, que se refere basicamente ao mesmo método, mas considerando uma série histórica mais longa e fontes de informação adicionais, como a Pesquisa Nacional por Amostra de Domicílios (PNAD).

Com vistas a assegurar a comparabilidade temporal com os dados censitários, foram selecionados os registros de óbitos presentes no SIM no período de 1o de agosto de 2009 a 31 de julho de 2010 por meio da ferramenta TABWIN do Departamento de Informática do SUS (DATASUS; http://www2. datasus.gov.br/DATASUS/index.php?area $=0901 \&$ item $=1 \&$ acao $=26$, acessado em 02/Abr/2013). Inicialmente, foram selecionados, por UF, os registros de óbito segundo as cinco categorias de cor ou raça, além da cor ou raça ignorada, nas seguintes faixas de idade: < 1 ano, 1-4, 5-14, 15-29, 30-44, 45-59, 60-69, 70-79 e 80 anos ou mais. Em seguida, em cada UF, procedeu-se a redistribuição proporcional das idades ignoradas segundo categorias de cor ou raça, sendo o passo seguinte a redistribuição dos óbitos com cor ou raça ignorada. Foram aplicados fatores de correção para a subnotificação de óbitos segundo UF (http://tabnet.datasus.gov.br/cgi/idb2012/a1802b.htm, acessado em 27/Set/2015).

Para compor os denominadores das TMI com base no SIM/SINASC, foram obtidas as frequências de nascidos vivos valendo-se do SINASC para o período de 1o de agosto de 2009 a 31 de julho de 2010, segundo UF (http://www2.datasus.gov.br/DATASUS/index.php?area=0901\&item=1\&a$\mathrm{cao}=28 \& \mathrm{pad}=31655$, acessado em 25/Mar/2014). Tal como nos óbitos, os nascidos vivos cuja cor ou raça era ignorada foram redistribuídos proporcionalmente entre todas as categorias. Adicionalmente, foram aplicados fatores de correção para a subnotificação de nascidos vivos (http://tabnet.datasus. gov.br/cgi/idb2012/a17b.htm, acessado em 27/Set/2015). 
Valendo-se dos procedimentos de análise dos dados de óbito e de nascidos vivos gerados pelo SIM/SINASC segundo UF, foram calculadas as TMI por cor ou raça para cada uma das macrorregiões do país.

Para o cálculo das TMI segundo componentes, foram obtidos para cada UF os registros de óbitos no SIM de 1o de janeiro de 2009 a 31 de dezembro de 2010 usando-se a ferramenta TABWIN (http://www2.datasus.gov.br/DATASUS/index.php?area=0901\&item =1\&acao=26, acessado em 25/ Fev/2015). Para se garantir que as frequências não se tornassem por demais rarefeitas ao se estratificar por cor ou raça e região, optou-se pelo período de dois anos de modo a ampliar o número de registros, o que aconteceu após a verificação de que o perfil geral dos dados no tocante à distribuição regional e por idade era semelhante em ambos. Foram empregadas as faixas de 0-6 dias (neonatal precoce), 7-27 dias (neonatal tardio) e 28-364 dias (pós-neonatal) e as cinco categorias de cor ou raça. Os óbitos de cor ou raça ignorados foram redistribuídos proporcionalmente entre as cinco categorias, segundo UF. Foram aplicados fatores de correção para a subnotificação de óbitos por UF (http://tabnet.datasus. gov.br/cgi/idb2012/a1802b.htm, acessado em 27/Set/2015).

Para compor os denominadores para cálculo das TMI segundo componentes, foram obtidas, por UF, as frequências de nascidos vivos oriundas do SINASC para o mesmo período de 24 meses anteriormente indicado (http://www2.datasus.gov.br/DATASUS/index.php?area=0901\&item=1\&a $\mathrm{cao}=28 \& \mathrm{pad}=31655$, acessado em 25/Fev/2015). Tal como nos óbitos, os nascidos vivos de cor ou raça ignorada foram redistribuídos proporcionalmente entre as cinco categorias. Assim como nos óbitos, foram aplicados fatores de correção para a subnotificação de nascidos vivos por UF (http:// tabnet.datasus.gov.br/cgi/idb2012/a17b.htm, acessado em 27/Set/2015). Os procedimentos de análise antes descritos foram conduzidos utilizando-se o pacote estatístico IBM SPSS, versão 20 (IBM Corp., Armonk, Estados Unidos) e software Excel 2010 (Microsoft Corp., Estados Unidos).

A presente pesquisa se baseia em bancos de dados disponibilizados pelo IBGE e pelo DATASUS, que não são de acesso restrito. Desse modo, não foi necessária a apreciação por um Comitê de Ética, conforme indicado na Resolução CNS/MS no 466 de 12 de dezembro de 2012.

\section{Resultados}

De acordo com a pergunta de óbitos no domicílio no Censo de 2010, ocorreram 35.055 óbitos em menores de um ano de idade (Tabela 1). Quanto ao SIM, o total foi de 48.950, ou seja, 39,6\% superior. As comparações entre as duas fontes indicaram proporções mais elevadas de óbitos no SIM para brancos, pardos e indígenas, com pretos e amarelos com proporções mais expressivas no IBGE.

O Censo de 2010 registrou 2.662.166 nascidos vivos, já a frequência segundo o SINASC foi de 3.022.242 (Tabela 1). Ou seja, houve uma captação 13,5\% maior. Quanto às diferenças entre as duas fontes, o Censo de 2010 captou proporcionalmente mais pretos, amarelos e indígenas, e registrou proporções menores de brancos e pardos.

Para o país como um todo, de acordo com os dois métodos para o cálculo da TMI empregados com base nos dados censitários, as mais baixas taxas foram observadas em brancos e amarelos, e as mais elevadas em indígenas (Tabela 2). Para o total da população do país, a TMI foi de 15,9/1.000 nascidos vivos utilizando-se os dados de óbitos, e de 17,2/1.000 pelo método dos filhos sobreviventes.

Quanto à TMI baseando-se nos dados do SIM/SINASC (16,3/1.000), também foram observados valores mais baixos para brancos e amarelos no país como um todo, já os indígenas apresentaram os mais elevados (47,2/1.000) (Tabela 3).

Em geral, ao se comparar as estimativas obtidas pelo Censo de 2010 pelos dois métodos empregados com aquelas derivadas do SIM/SINASC (Tabelas 2 e 3), as TMI de brancos (13,8; 15,4 e 15,2), pardos $(16,9 ; 18,2$ e 16,6) e a população em geral (15,9; 17,2 e 16,3) estiveram relativamente próximas em cada um destes grupos de cor ou raça e no Brasil em geral. Por outro lado, para as demais categorias de cor ou raça, houve diferenças expressivas. No caso dos amarelos, a TMI com base no SIM/ SINASC (9,7/1.000) foi bem menor do que as derivadas do Censo de 2010 (15,4 e 14,5/1.000). Pretos e indígenas, por sua vez, apresentaram TMI baseando-se nos dados do SIM/SINASC bastante superiores (29,1 e 47,2/1.000, respectivamente). 


\section{Tabela 1}

Frequências de óbitos em crianças menores de um ano e de nascidos vivos por cor ou raça segundo o Instituto Brasileiro de Geografia e Estatística (IBGE) e o Departamento de Informática do SUS (DATASUS). Brasil, agosto de 2009 a julho de 2010.

\begin{tabular}{|c|c|c|c|c|c|c|c|c|}
\hline \multirow[t]{3}{*}{ Cor ou raça } & \multicolumn{4}{|c|}{ IBGE } & \multirow{2}{*}{\multicolumn{2}{|c|}{$\begin{array}{c}\text { SIM } \\
\text { Óbitos }\end{array}$}} & \multirow{2}{*}{\multicolumn{2}{|c|}{$\begin{array}{c}\text { SINASC } \\
\text { Nascidos vivos }\end{array}$}} \\
\hline & \multicolumn{2}{|c|}{ Óbitos } & \multicolumn{2}{|c|}{ Nascidos vivos } & & & & \\
\hline & $\mathbf{n}$ & $\%$ & $\mathbf{n}$ & $\%$ & $\mathbf{n}$ & $\%$ & $\mathbf{n}$ & $\%$ \\
\hline Branca & 12.516 & 35,7 & 1.091 .696 & 41,0 & 20.794 & 42,5 & 1.365 .773 & 45,5 \\
\hline Preta & 4.185 & 11,9 & 267.538 & 10,0 & 1.329 & 2,7 & 45.720 & 1,5 \\
\hline Amarela & 389 & 1,1 & 30.430 & 1,1 & 59 & 0,1 & 6.063 & 0,2 \\
\hline Parda & 17.495 & 49,9 & 1.251 .770 & 47,0 & 25.965 & 53,0 & 1.567 .678 & 52,2 \\
\hline Indígena & 470 & 1,3 & 20.732 & 0,8 & 803 & 1,6 & 17.008 & 0,6 \\
\hline Total & 35.055 & 100,0 & 2.662 .166 & 100,0 & 48.950 & 100,0 & 3.022 .242 & 100,0 \\
\hline
\end{tabular}

SIM: Sistema de Informação sobre Mortalidade; SINASC: Sistema de Informaç̃es sobre Nascidos Vivos.

Fontes: IBGE (Censo Demográfico de 2010) e DATASUS (SIM e SINASC, 2009-2010).

\section{Tabela 2}

Frequências de óbitos, nascidos vivos e estimativas de mortalidade infantil segundo o Censo Demográfico de 2010 de acordo com a cor ou raça. Brasil, 2010.

\begin{tabular}{|c|c|c|c|c|}
\hline \multirow[t]{2}{*}{ Cor ou raça } & \multicolumn{2}{|c|}{ Censo de 2010} & \multicolumn{2}{|c|}{ TMI } \\
\hline & Óbitos nos domicílios * & Filhos nascidos vivos nos últimos 12 meses ** & $\star * *$ & $\#$ \\
\hline Branca & 12.516 & 1.091 .696 & 13,8 & 15,4 \\
\hline Preta & 4.185 & 267.538 & 18,9 & 17,7 \\
\hline Amarela & 389 & 30.430 & 15,4 & 14,5 \\
\hline Parda & 17.495 & 1.251 .770 & 16,9 & 18,2 \\
\hline Indígena & 470 & 20.732 & 27,3 & 21,5 \\
\hline Total & 35.055 & 2.662 .166 & 15,9 & 17,2 \\
\hline
\end{tabular}

TMI: taxas de mortalidade infantil.

* Óbitos menores de um ano no domicílio por cor ou raça do responsável;

** Filho tido nascido vivo no período de referência de 12 meses anteriores a 31 de julho de 2010, de acordo com a cor ou raça do responsável;

*** Taxas corrigidas pelos fatores de ajuste propostos por Queiroz \& Sawyer 20;

\# Método dos filhos sobreviventes diretamente pelo Censo de 2010, ajustado para o total do Brasil divulgado pelo Instituto Brasileiro de Geogafia e Estatística (IBGE) 19.

Fonte: Censo Demográfico de 2010 (IBGE).

De acordo com os dados do SIM/SINASC, dentre todas as categorias de cor ou raça, os indígenas apresentaram as mais elevadas TMI nas regiões Sul, Sudeste e Centro-oeste, alcançando 56,7/1.000 nesta última (Tabela 3). No Norte e Nordeste, as taxas mais elevadas foram observadas em pretos (52,5 e 50,1/1.000, respectivamente), com os indígenas na segunda posição. A categoria amarela apresentou as menores taxas em todas as regiões, com exceção do Norte, onde foi observado um valor surpreendentemente elevado (28,8/1.000). No Norte e Nordeste, as TMI de pardos estiveram mais baixas do que as de brancos. Chama atenção que, com exceção do Sul, as TMI para pardos estiveram mais próximas daquelas de brancos, com destaque para o Centro-oeste e o Sudeste, do que daquelas de pretos ou indígenas. Com exceção dos indígenas, com a elevada TMI de 29,7/1.000, foi no Sul que se observaram as menores diferenças nas TMI dentre as demais categorias de cor ou raça, variando de 6,8/1.000 em amarelos a 11,9/1.000 em pardos.

Especificamente quanto aos indígenas, as TMI, ainda que bastante elevadas (em geral > 30,0/1.000), apresentaram variações regionais expressivas (Tabela 3). Os valores foram mais baixos no Sul e 
Tabela 3

Taxas de mortalidade infantil (TMI) por cor ou raça segundo o Sistema de Informação sobre Mortalidade (SIM) e o Sistema de Informações sobre Nascidos Vivos (SINASC). Brasil e regiões, agosto de 2009 a julho de 2010.

\begin{tabular}{|c|c|c|c|c|c|c|}
\hline \multirow[t]{2}{*}{ Região } & \multicolumn{6}{|c|}{ Cor ou raça (TMI *) } \\
\hline & Branca & Preta & Amarela & Parda & Indígena & Total \\
\hline Norte & 29,9 & 52,5 & 28,0 & 18,8 & 50,7 & 21,5 \\
\hline Nordeste & 26,5 & 50,1 & 8,8 & 17,8 & 37,5 & 19,7 \\
\hline Sudeste & 13,3 & 24,1 & 8,1 & 13,6 & 31,1 & 13,6 \\
\hline Sul & 11,5 & 11,4 & 6,8 & 11,9 & 29,7 & 11,6 \\
\hline Centro-oeste & 16,7 & 43,2 & 6,3 & 14,4 & 56,7 & 16,2 \\
\hline Brasil & 15,2 & 29,1 & 9,7 & 16,6 & 47,2 & 16,3 \\
\hline
\end{tabular}

* Taxas por 1.000 nascidos vivos.

Fonte: Departamento de Informática do SUS (SIM e SINASC, 2010).

Sudeste, e superaram 50,0/1.000 no Norte e no Centro-oeste. Para o Brasil como um todo, a TMI de indígenas foi aproximadamente três vezes superior ao valor nacional (47,2/1.000 vs. 16,3/1.000). Em todas as regiões, as taxas para os indígenas foram aproximadamente o dobro daquelas do total da população, alcançando 3,5 vezes no Centro-oeste.

Quanto à mortalidade proporcional segundo componentes, nas regiões e no país como um todo observou-se que mais da metade dos óbitos estão no período neonatal precoce (Tabela 4). Nota-se que, ao se considerar conjuntamente a mortalidade neonatal precoce e tardia $(<28$ dias), as proporções para todas as categorias de cor e raça nas diversas regiões superam os 60\%, com exceção de amarelos (somente no Centro-oeste) e indígenas (em todas as regiões). Em todas as regiões, as proporções de óbitos no componente pós-neonatal se mostraram expressivamente mais elevadas nos indígenas (> $50 \%)$, se comparadas às demais categorias de cor ou raça, em geral 30-40\% menores. A única exceção é aquela dos amarelos no Centro-oeste, com 67,9\% dos óbitos no período pós-neonatal.

O período neonatal precoce foi o que apresentou as taxas mais elevadas no Brasil como um todo $(8,7 / 1.000)$ e em todas as regiões, com destaque para o Norte $(11,3 / 1.000)$ e o Nordeste $(11 / 1.000)$ (Tabela 5). O neonatal tardio apresentou as menores taxas dentre os três componentes $(2,6 / 1.000)$. Quanto ao componente pós-neonatal, foi observada uma taxa de 5,1/1.000 no país como um todo, com Norte e Nordeste apresentando valores ligeiramente superiores àqueles das demais regiões.

Para os componentes da mortalidade infantil, os indígenas apresentaram as mais elevadas taxas ao se considerar o país como um todo (13,6, 4,8 e 29,1/1.000) (Tabela 5). No caso dos componentes neonatal precoce e tardio, com poucas exceções, indígenas e pretos tenderam a apresentar os valores mais elevados ao se comparar as regiões, já amarelos e brancos os mais baixos.

Ainda no tocante aos componentes, as diferenças entre indígenas e demais categorias de cor ou raça se mostraram particularmente acentuadas para o período pós-neonatal (Tabela 5). Assim, as razões entre as TMI de indígenas em relação ao total da população variaram de 3,5 a 5,6 no Nordeste e Centro-oeste, respectivamente. Essas razões são superiores àquelas TMI de pretos em relação à população como um todo de cada uma das regiões, que variaram de 1,7 no Centro-oeste a 2,8 no Nordeste.

\section{Discussão}

A inclusão de perguntas no Censo de 2010 acerca da ocorrência de óbitos nos domicílios foi uma importante iniciativa com vistas a um melhor conhecimento quanto aos perfis de mortalidade no país 20. Dentre outros aspectos, tem o potencial de permitir o aprofundamento das análises sobre a mortalidade infantil segundo cor ou raça. Se comparados a outras fontes, em particular o SIM e o SINASC, os dados censitários podem ser utilizados para investigar separadamente contextos urbanos e rurais, 
Tabela 4

Proporções de óbitos por faixas de idade, segundo cor ou raça. Brasil e regiões, 2009-2010.

\begin{tabular}{llcccccc}
\hline \multirow{2}{*}{ Faixa etária (dias) } & Regiões & & \multicolumn{5}{c}{ Cor ou raça (\%) } \\
& & Branca & Preta & Amarela & Parda & Indígena & Total \\
\hline \multirow{2}{*}{0 -6 } & Norte & 45,3 & 46,9 & 39,0 & 56,6 & 25,9 & 52,0 \\
& Nordeste & 47,0 & 48,3 & 62,8 & 59,5 & 40,7 & 56,2 \\
& Sudeste & 50,1 & 45,2 & 43,7 & 53,6 & 33,1 & 51,1 \\
& Sul & 51,4 & 53,3 & 39,7 & 46,1 & 28,8 & 51,0 \\
& Centro-oeste & 49,1 & 56,7 & 32,1 & 54,5 & 28,5 & 50,9 \\
& Brasil & 49,3 & 47,6 & 47,3 & 57,2 & 28,7 & 53,1 \\
& Norte & 15,8 & 13,0 & 21,3 & 15,0 & 9,9 & 14,8 \\
& Nordeste & 15,1 & 12,5 & 7,1 & 13,6 & 4,1 & 13,9 \\
& Sudeste & 18,9 & 17,4 & 22,1 & 16,4 & 8,9 & 17,9 \\
& Sul & 18,1 & 18,8 & 20,3 & 19,5 & 11,6 & 18,2 \\
& Centro-oeste & 18,6 & 13,0 & 0,0 & 16,1 & 14,2 & 17,0 \\
& Brasil & 17,7 & 15,2 & 15,5 & 14,7 & 10,0 & 15,9 \\
& Norte & 38,9 & 40,1 & 39,7 & 28,4 & 64,2 & 33,2 \\
& Nordeste & 37,9 & 39,2 & 30,1 & 26,9 & 55,2 & 29,9 \\
& Sudeste & 31,0 & 37,4 & 34,2 & 30,1 & 58,0 & 30,9 \\
& Sul & 30,5 & 27,9 & 40,0 & 34,4 & 59,6 & 30,8 \\
& Centro-oeste & 32,4 & 30,3 & 67,9 & 29,4 & 57,3 & 30,2 \\
& Brasil & 33,0 & 37,2 & 37,2 & 28,1 & 61,3 & 31,0 \\
\hline
\end{tabular}

Fonte: Departamento de Informática do SUS (SIM e SINASC, 2010).

uma modalidade de desagregação que não está disponível nessas outras bases ${ }^{7}$. Além disso, permite o cálculo da mortalidade infantil com numerador e denominador oriundos da mesma fonte de dados (ou seja, o censo demográfico), o que evita questões relativas à incompatibilidade de definições e forma de declaração em fontes distintas.

Até o momento, poucas análises sobre mortalidade no país foram realizadas utilizando dados do Censo de 2010, com destaque para o trabalho de Queiroz \& Saywer 20. Em linhas gerais, esses autores indicaram que a qualidade da informação é elevada, de modo que constituem "uma ferramenta muito útil para estudos de mortalidade no Brasil, em especial para estudar diferenciais de mortalidade em pequenas áreas” 20 (p. 235). Vale mencionar que a principal questão abordada por Queiroz \& Sawyer 20 foi a estrutura etária, não tendo contemplado aspectos como estratificação segundo regiões do país ou cor ou raça.

Com foco na mortalidade infantil, o presente estudo se alinha com a ênfase acerca da importância dos dados do Censo de 2010 para fins de pesquisas sobre esta mortalidade no país. Por um lado, foram observadas diferenças nos montantes de captação de óbitos e de nascidos vivos ao se comparar o Censo de 2010 e o SIM/SINASC, com valores mais baixos para o levantamento censitário. Não obstante, chama atenção a proximidade dos valores da taxa de mortalidade infantil ao se comparar os dados censitários com aqueles derivados dos sistemas de informação (SIM e SINASC). Cobrindo o mesmo período de tempo, de agosto de 2009 a julho de 2010, foram obtidas TMI bastante próximas por meio das fontes de dados utilizadas. No caso dos dados censitários, os dois métodos empregados (direto e filhos sobreviventes) chegaram a valores de 15,9 e 17,2/1.000 nascidos vivos, e a taxa foi de 16,3/1.000 baseando-se no SIM/SINASC.

Se nas estimativas de mortalidade infantil no plano mais geral do país se observou concordância entre os dados do Censo de 2010 e aqueles do SIM/SINASC, análises desagregadas evidenciaram taxas que, por vezes, se mostram discordantes. Nesse sentido, verificou-se que as TMI de indígenas derivadas tanto dos dados do Censo de 2010 como do SIM/SINASC tenderam a ser mais elevadas do 


\section{Tabela 5}

Taxas de mortalidade infantil (TMI) por componentes, segundo cor ou raça. Brasil e regiões, 2009-2010.

\begin{tabular}{|c|c|c|c|c|c|c|c|}
\hline \multirow[t]{2}{*}{ Faixa etária (dias) } & \multirow[t]{2}{*}{ Regiões } & \multicolumn{6}{|c|}{ Cor ou raça (TMI *) } \\
\hline & & Branca & Preta & Amarela & Parda & Indígena & Total \\
\hline \multirow[t]{6}{*}{$0-6$} & Norte & 14,1 & 21,1 & 14,6 & 10,7 & 13,7 & 11,3 \\
\hline & Nordeste & 12,3 & 20,5 & 8,7 & 10,6 & 15,2 & 11,0 \\
\hline & Sudeste & 6,6 & 10,3 & 3,7 & 7,3 & 11,9 & 6,9 \\
\hline & Sul & 5,9 & 6,2 & 3,3 & 5,5 & 7,4 & 5,9 \\
\hline & Centro-oeste & 8,2 & 16,0 & 3,8 & 8,0 & 14,6 & 8,2 \\
\hline & Brasil & 7,5 & 12,7 & 6,0 & 9,5 & 13,6 & 8,7 \\
\hline \multirow[t]{6}{*}{$7-27$} & Norte & 4,9 & 5,9 & 8,0 & 2,8 & 5,2 & 3,2 \\
\hline & Nordeste & 4,0 & 5,3 & 1,0 & 2,4 & 1,5 & 2,7 \\
\hline & Sudeste & 2,5 & 4,0 & 1,9 & 2,2 & 3,2 & 2,4 \\
\hline & Sul & 2,1 & 2,2 & 1,7 & 2,3 & 3,0 & 2,1 \\
\hline & Centro-oeste & 3,1 & 3,7 & 0,0 & 2,4 & 7,3 & 2,8 \\
\hline & Brasil & 2,7 & 4,1 & 2,0 & 2,4 & 4,8 & 2,6 \\
\hline \multirow[t]{6}{*}{$28-364$} & Norte & 12,1 & 18,0 & 14,9 & 5,4 & 34,0 & 7,2 \\
\hline & Nordeste & 10,0 & 16,6 & 4,1 & 4,8 & 20,6 & 5,9 \\
\hline & Sudeste & 4,1 & 8,5 & 2,9 & 4,1 & 20,8 & 4,2 \\
\hline & Sul & 3,5 & 3,2 & 3,3 & 4,1 & 15,3 & 3,6 \\
\hline & Centro-oeste & 5,4 & 8,6 & 8,1 & 4,3 & 29,2 & 5,2 \\
\hline & Brasil & 5,0 & 9,9 & 4,7 & 4,7 & 29,1 & 5,1 \\
\hline
\end{tabular}

* Taxas por 1.000 nascidos vivos.

Fonte: Departamento de Informática do SUS (SIM e SINASC, 2010).

que aquelas para os demais segmentos da população brasileira. Além disso, se aproximaram daquelas de outros segmentos reconhecidamente desfavorecidos, como é o caso dos pretos, confirmando padrões de desigualdade étnico-racial que vêm sendo descritos em diversas investigações em demografia e saúde coletiva realizadas no país 7,8,9,10. Entretanto, ainda que essas desigualdades de cor ou raça se confirmem por meio dos dados censitários, as TMI calculadas com base no Censo de 2010 para indígenas e pretos se mostraram bastante baixas se comparadas às derivadas do SIM/SINASC. Mesmo com diferenças em magnitude, o padrão geral de menores valores de TMI em brancos e amarelos e maiores em pretos e indígenas foi evidenciado tanto nas análises do Censo de 2010 como nas do SIM/SINASC.

Particularidades metodológicas no que diz respeito à coleta dos dados no Censo de 2010 e no SIM/SINASC devem ser levadas em consideração na interpretação de diferenças em níveis de mortalidade infantil segundo cor ou raça no Brasil. Os dados censitários são obtidos por meio de entrevistas realizadas nas residências, com a atribuição da cor ou raça feita pela própria pessoa ou por outro informante 19. Desse modo, ao prover informação sobre si próprio ao recenseador, o entrevistado se autoclassifica quanto à cor ou raça, já os demais moradores são heteroclassificados (ou seja, a cor ou raça não é autoatribuída). Acrescente-se que, no caso dos óbitos, conforme já sinalizado, não foram coletados dados sobre cor ou raça no Censo de 2010, tendo sido a estratégia metodológica do presente estudo atribuir a cor ou raça do responsável pelo domicílio. Já no caso do SIM, as informações são oriundas da Declaração de Óbito (DO). Segundo as orientações do Ministério da Saúde, para o preenchimento da DO a cor ou raça do falecido deve ser primordialmente oriunda de informações prestadas por pessoa da relação com o morto, somente devendo ser atribuída pelo médico com base na observação na ausência desta outra fonte 24. Até 2011, a classificação da cor ou raça do recém-nascido no SINASC era feita pelo profissional de saúde responsável pelo preenchimento da Declaração de Nascido Vivo 25,26. 
Desse modo, é possível que, em parte, as diferenças nas TMI segundo as categorias de cor ou raça observadas neste estudo possam em parte estar associadas às particularidades dos procedimentos de coleta de dados no Censo de 2010 e no SIM/SINASC. Apesar de termos realizado uma detalhada revisão bibliográfica, não identificamos análises na literatura que explorem os fatores que poderiam explicar as diferenças observadas entre essas fontes. É um tema relevante que, por certo, merece atenção de pesquisadores da área da saúde coletiva e demografia interessados no estudo da mortalidade segundo cor ou raça.

Vale indicar que questões acerca das limitações do uso da variável cor ou raça nos estudos de saúde têm sido apontadas por diversos autores, no Brasil e no exterior 7,8,12,27,28,29. Segundo Cardoso et al. 7, há que se considerar as deficiências na cobertura e qualidade dos sistemas nacionais de informação em saúde. Além disso, tem sido sugerido que a forma mais adequada de classificação, seja autoclassificação, classificação por terceiros (heteroclassificação) ou outras modalidades (como a utilização de métodos combinados), depende dos objetivos específicos das investigações 30,31.

Nas duas últimas décadas tem ocorrido uma significativa expansão dos estudos sobre demografia e saúde dos povos indígenas no Brasil 7,14,32,33. Em 2008-2009, foi realizado o I Inquérito Nacional de Saúde e Nutrição dos Povos Indígenas, cuja amostra envolveu crianças menores de cinco anos de mais de uma centena de aldeias de todo o país ${ }^{32}$. Ainda que não tenham sido produzidas informações acerca de mortalidade infantil, os resultados desse Inquérito apontaram para importantes desigualdades em indicadores de saúde ao se comparar indígenas e não indígenas no país. Desse modo, ficou evidenciado que as crianças indígenas apresentaram maiores prevalências de baixa estatura e peso para idade e de anemia, assim como maior ocorrência de diarreia e infecção respiratória aguda (IRA) ${ }^{32}$. Nesse contexto, os mais elevados níveis de mortalidade infantil em indígenas observados no presente trabalho são consistentes com os cenários de desigualdade detectados em estudos sobre as condições de vida de crianças indígenas em comparação com as não indígenas no país.

A partir de 1999, com a implantação do Subsistema de Atenção à Saúde dos Povos Indígenas, foi criado o Sistema de Informação da Atenção à Saúde Indígena (SIASI) 34. Ainda que seja amplamente reconhecido que o SIASI apresente problemas de cobertura e completitude 35, assim como deve ser mencionado que a população atendida seja, sobretudo, a residente em aldeias, é a única outra fonte de informação, além do Censo e do SIM/SINASC, sobre a mortalidade infantil em indígenas de abrangência nacional. Nos anos recentes, não têm sido divulgadas estimativas de TMI para indígenas com base nos dados do SIASI. As estimativas mais recentes são de 2009, com uma TMI de 41,9/1.000 nascidos vivos. De 2000 a 2009, houve uma tendência de queda de 43,8\% 36. Portanto, em termos de magnitude, observa-se uma proximidade da TMI entre o SIASI e as fontes detalhadas neste estudo, a despeito de todas as diferenças no tocante à população coberta e procedimentos de coleta e análise de dados sobre mortalidade infantil.

O padrão de mortalidade infantil por componentes observado no Brasil, em particular no que diz respeito à importância da proporção do componente neonatal, tem sido também observado em outros países. Um estudo de revisão sistemática sobre causas globais, regionais e nacionais de mortalidade infantil com análises de tendência entre 2000 e 2010 em países da África, das Américas, Europa, Leste Europeu, Sudoeste da Ásia, Austrália e Oceania identificou que, de aproximadamente 7,6 milhões de mortes em menores de cinco anos de idade, 40\% ocorreram no período neonatal 37.

Tal como no presente estudo, a concentração dos óbitos no componente pós-neonatal foi observada em outros trabalhos com a população indígena no Brasil e nos Estados Unidos. Tomashek et al. 38 reportaram mudanças nas taxas de mortalidade infantil por idade e por causas em populações indígenas nos Estados Unidos, em comparação com crianças brancas. Nos dois períodos analisados por esses autores (1989-1991 e 1998-2000), as TMI declinaram em indígenas e não indígenas, mas a TMI em indígenas esteve 1,7 vez maior, sendo a diferença sobretudo devido à elevada mortalidade pós-neonatal 38. Em uma das poucas investigações que abordaram os componentes da mortalidade infantil em crianças indígenas no Brasil, Cardoso et al. 39 indicaram uma TMI pós-neonatal bastante elevada (24,7/1.000) nos Guarani do Sul e Sudeste do Brasil.

Quanto às limitações deste trabalho, cabe destacar o critério de classificação de cor ou raça do óbito nos dados censitários, tendo sido alocada a mesma do responsável pelo domicílio. Análises recentes têm explorado aspectos ligados à composição de domicílios segundo cor ou raça, evidenciando-se que a depender de fatores socioeconômicos e geográficos, incluindo situação urbana e rural, 
pode haver variação nas proporções de domicílios homogêneos para a característica 40 . No caso dos dados do Censo de 2010, uma importante linha de análise seriam investigações comparativas dos níveis de mortalidade segundo cor ou raça empregando-se, além da característica do responsável, outros critérios de classificação como, por exemplo, atribuir ao óbito a mesma cor ou raça da maioria dos moradores.

\section{Considerações finais}

Conforme apontado por Victora et al. 3, a mortalidade na infância decresceu acentuadamente no Brasil nas últimas três décadas, o que largamente é atribuído ao fato de que a saúde infantil passou a ser prioridade na agenda política do país. Nesse período, as desigualdades regionais também diminuíram, assim como as disparidades sociais, embora em menor grau. Decréscimos substancialmente mais pronunciados foram observados para algumas causas de morte, com destaque para as doenças infecciosas. A melhoria dos indicadores de saúde infantil no Brasil nas últimas décadas não aconteceu somente pelo aumento de cobertura dos serviços de saúde, como também pela melhoria nas condições de renda e educação. Não obstante, com base no evidenciado no presente estudo, o impacto das políticas públicas não alcançou os povos indígenas no país, cuja mortalidade infantil permanece em níveis bastante mais elevados se comparada a outros segmentos da população brasileira. Como se buscou abordar neste trabalho, novas fontes de dados relativos à ocorrência de óbitos nos domicílios, de abrangência nacional, como é o caso do Censo de 2010, podem contribuir para a melhor compreensão das desigualdades segundo cor ou raça.

\section{Colaboradores}

A. D. R. Caldas participou da concepção do projeto, análise dos dados, interpretação dos resultados, redação do manuscrito e revisão do texto final. $\mathrm{R}$. V. Santos participou da concepção do projeto, discussão dos resultados, redação e revisão do texto final. G. M. Borges, J. G. Valente, M. C. Portela e G. L. Marinho contribuíram com a análise dos dados, redação, revisão crítica do manuscrito e aprovação final da versão a ser publicada.

\section{Agradecimentos}

Agradecimentos à Fundação Carlos Chagas Filho de Amparo à Pesquisa do Estado do Rio de Janeiro (Faperj; E-26/102.352/2013 - Bolsa "Cientista de Nosso Estado"). 


\section{Referências}

1. Duarte CMR. Reflexos das políticas de saúde sobre as tendências da mortalidade infantil no Brasil: revisão da literatura sobre a última década. Cad Saúde Pública 2007; 23:1511-28.

2. Departamento de Análise de Situação em Saúde, Secretaria de Vigilância em Saúde, Ministério da Saúde. Saúde Brasil 2013: uma análise da situação de saúde e das doenças transmissíveis relacionadas à pobreza. Brasília: Ministério da Saúde; 2014.

3. Victora CG, Aquino EML, Leal MC, Monteiro CA, Barros FC, Szwarcwald CL. Maternal and child health in Brazil: progress and challenges. Lancet 2011; 377:1863-76.

4. França E, Lansky S. Mortalidade infantil neonatal no Brasil: situação, tendências e perspectivas. http://www.abep.nepo.unicamp.br/ encontro2008/docsPDF/ABEP2008_1956.pdf (acessado em 25/Nov/2015).

5. Boing AF, Boing AC. Mortalidade infantil por causas evitáveis no Brasil: um estudo ecológico no período 2000-2002. Cad Saúde Pública 2008; 24:447-55.

6. Carneiro RM, Franco RA, Medeiros RM, Schwab GL. Mortalidade infantil e estratificação social: subsídios para a elaboração de políticas de saúde. Arq Catarin Med 2012; 41:47-50.

7. Cardoso AM, Santos RV, Coimbra Jr. CEA. Mortalidade infantil segundo raça/cor no Brasil: o que dizem os sistemas nacionais de informação? Cad Saúde Pública 2005; 21:1602-8.

8. Chor D, Lima CRA. Aspectos epidemiológicos das desigualdades raciais em saúde no Brasil. Cad Saúde Pública 2005; 21:1586-94.

9. Matijasevich A, Victora CG, Barros AJD, Santos IS, Marco PL, Albernaz EP, et al. Widening ethnic disparities in infant mortality in southern Brazil: comparison of 3 birth cohorts. Am J Public Health 2008; 98:692-8.

10. Wong LLR, Barros JS, Santos W. Mortalidad infantil e infanto juvenil en Brasil según sexo y color de la piel. In: Wong LLR, Sánchez JA, organizadores. Situación de la población afrodescendiente e indígena en América Latina puntos de reflexión para el debate sobre Cairo+20. Belo Horizonte: Asociación Latinoamericana de Población; 2014. p. 79-98.

11. Simões CCS. Perfis de saúde e mortalidade no Brasil: uma análise de seus condicionantes em grupos populacionais específicos. Brasília: Organização Pan-Americana da Saúde; 2002.
12. Travassos C, Williams DR. The concept and measurement of race and their relationship to public health: a review focused on Brazil and the United States. Cad Saúde Pública 2004; 20:660-78.

13. Drumond E, Abreu DM, Machado C, Gomes F, França E. Racial disparities and avoidable infant mortality in a city of southeastern Brazil, 2001-09. J Trop Pediatr 2013; 59:23-8.

14. Pagliaro H, Azevedo MM, Santos RV, organizadores. Demografia dos povos indígenas no Brasil. Rio de Janeiro: Editora Fiocruz/Associação Brasileira de Estudos Populacionais; 2005.

15. Santos RV, Teixeira P. O "indígena" que emerge do Censo Demográfico de 2010. Cad Saúde Pública 2011;27:1048-9.

16. Instituto Brasileiro de Geografia e Estatística. Tendências demográficas: uma análise dos indígenas com base nos resultados da amostra dos Censos Demográficos 1991 e 2000. Rio de Janeiro: Instituto Brasileiro de Geografia e Estatística; 2005.

17. Instituto Brasileiro de Geografia e Estatística. Censo Demográfico de 2010. Características gerais dos indígenas. Resultados do universo. Rio de Janeiro: Instituto Brasileiro de Geografia e Estatística; 2012.

18. Instituto Brasileiro de Geografia e Estatística. Censo Demográfico de 2010. Características da população e dos domicílios. Resultados do universo. Rio de Janeiro: Instituto Brasileiro de Geografia e Estatística; 2011.

19. Instituto Brasileiro de Geografia e Estatística. Metodologia do Censo Demográfico de 2010. Rio de Janeiro: Instituto Brasileiro de Geografia e Estatística; 2013. (Série Relatórios Metodológicos, 41).

20. Queiroz BL, Sawyer DOT. O que os dados de mortalidade do Censo de 2010 podem nos dizer? Rev Bras Estud Popul 2012; 29:225-38.

21. Brass W, Coale AJ. Methods of analysis and estimation. In: Brass W, Coale AJ, Demeny P, Heisel DF, editors. The demography of tropical Africa. Princeton: Princeton University Press; 1968. p. 127-32.

22. Coale AJ, Trussell J. Annex I: estimating the time to which Brass estimates apply. Popul Bull UN 1977; 10:87-9. 
23. Instituto Brasileiro de Geografia e Estatística. Projeção da população do Brasil por sexo e idade para o período 2000-2060. Projeção da população das Unidades da Federação por sexo e idade para o período de 2000-2030. ftp:// ftp.ibge.gov.br/Projecao_da_Populacao/Pro jecao_da_Populacao_2013/nota_metodologi ca_2013.pdf (acessado em 06/Jul/2016).

24. Departamento de Análise de Situação de Saúde, Secretaria de Vigilância em Saúde, Ministério da Saúde. Manual de instruções para o preenchimento da Declaração de Óbito. Brasília: Ministério da Saúde; 2011. (Série A. Normas e Manuais Técnicos).

25. Ministério da Saúde. Manual de procedimentos do Sistema de Informações sobre Nascidos Vivos. Brasília: Fundação Nacional de Saúde; 2001.

26. Departamento de Análise de Situação de Saúde, Secretaria de Vigilância em Saúde, Ministério da Saúde. Manual de instruções para o preenchimento da Declaração de Nascidos Vivos. Brasília: Ministério da Saúde; 2011. (Série A. Normas e Manuais Técnicos).

27. Instituto Brasileiro de Geografia e Estatística. Características étnico-raciais da população: um estudo das categorias de cor ou raça, 2008. Rio de Janeiro: Instituto Brasileiro de Geografia e Estatística; 2011.

28. Mello-Jorge MHP, Laurenti R, Gotlieb SLD. Análise da qualidade das estatísticas vitais brasileiras: a experiência de implantação do SIM e do SINASC. Ciênc Saúde Coletiva 2007; 12:643-54

29. Chor D. Desigualdades em saúde no Brasil: é preciso ter raça. Cad Saúde Pública 2013; 29:1272-5

30. Maio MC, Monteiro S, Chor D, Faerstein E, Lopes CS. Cor/raça no Estudo Pró-Saúde: resultados comparativos de dois métodos de autoclassificação no Rio de Janeiro, Brasil. Cad Saúde Pública 2005; 21:171-80.

31. Bastos JL, Peres MA, Peres KG, Dumith SC, Gigante DP. Diferenças socioeconômicas entre autoclassificação e heteroclassificação de cor/ raça. Rev Saúde Pública 2008; 42:324-34.
32. Coimbra Jr. CEA, Santos RV, Welch JR, Cardoso AM, Souza MC, Garnelo L, et al. The First National Survey of Indigenous People's Health and Nutrition in Brazil: rationale, methodology, and overview of results. BMC Public Health 2013; 13:1-19.

33. Montenegro RA, Stephens C. Indigenous health in Latin America and the Caribbean. Lancet 2006; 367:1859-69.

34. Ministério da Saúde; Fundação Nacional de Saúde. Política Nacional de Atenção à Saúde dos Povos Indígenas. Brasília: Ministério da Saúde; 2000.

35. Sousa MC, Scatena JHG, Santos RV. O Sistema de Informação da Atenção à Saúde Indígena (SIASI): criação, estrutura e funcionamento. Cad Saúde Pública 2007; 23:853-61.

36. Ministério da Saúde; Fundação Nacional de Saúde. Relatório de gestão, 2010 http://www.funasa.gov.br/site/wp-content/ uploads/2011/10/relatorio_2010.pdf (acessado em 19/Fev/2016)

37. Liu L, Johnson HL, Cousens S, Perin J, Scott S, Lawn JE, et al. Global, regional, and national causes of child mortality: an updated systematic analysis for 2010 with time trends since 2000. Lancet 2012; 379:2151-61.

38. Tomashek KM, Qin C, Hsia J, Iyasu S, Barfield WD, Flowers LM. Infant mortality trends and differences between American Indian/Alaska native infants and white infants in the United States, 1989-1991 and 1998-2000. Am J Public Health 2006; 96:2222-7.

39. Cardoso AM, Coimbra Jr. CEA, Barreto CTG, Werneck GL, Santos RV. Mortality among Guarani Indians in Southeastern and Southern Brazil. Cad Saúde Pública 2011; 27 Suppl 2: S222-36.

40. Marinho GL. Domicílios indígenas no Censo Demográfico de 2010: classificação, composição e interfaces com a saúde [Tese de Doutorado]. Rio de Janeiro: Escola Nacional de Saúde Pública Sergio Arouca, Fundação Oswaldo Cruz; 2015. 


\section{Abstract}

The aim of this study was to investigate infant mortality data according to color or race in $\mathrm{Bra}$ zil with a focus on indigenous individuals, based on data from the 2010 Population Census and the Brazilian Mortality Information System (SIM) and Brazilian Information System on Live Births (SINASC). In both sources, the infant mortality rate (IMR) for indigenous individuals was the highest of all the various population segments. Although the census data indicate inequalities by color or race, the infant mortality rates for indigenous and black individuals were lower than those based on data from SIM/SINASC. Methodological specificities in the data collection in the two sources should be considered. The reduction in IMR in Brazil in recent decades is largely attributed to the priority of infant health on the policy agenda. The study's findings indicate that the impact of public policies failed to reach indigenous peoples on the same scale as in the rest of the population. New sources of nationwide data on deaths in households, as in the case of the 2010 Census, can contribute to a better understanding of inequalities by color or race in Brazil.

South Americans Indians; Health of Indigenous Peoples; Infant Mortality; Health Inequalities

\section{Resumen}

El objetivo de este estudio es investigar la información sobre mortalidad infantil en Brasil, de acuerdo con el color de piel o raza, enfocándose en los indígenas, basándose en los datos del Censo Demográfico de 2010 y de aquellos oriundos del Sistema de Información sorbe Mortalidad (SIM) y del Sistema de Información sobre Nacidos Vivos (SINASC). En ambas fuentes, las tasas de mortalidad infantil (TMI) de indígenas fueron las más elevadas entre los diversos segmentos poblacionales. A pesar de los dados que indicaron desigualdades de color o raza, las TMI para indígenas y negros fueron inferiores a las derivadas del SIM/ SINASC. Particularidades metodológicas, referentes a la recogida de datos en ambas fuentes, deben ser consideradas. A la prioridad de la salud infantil en la agenda política se le atribuye en gran medida la reducción de la TMI en Brasil en las últimas décadas. Los hallazgos de este trabajo son indicativos de que el impacto de las politicas públicas no alcanzó a los indígenas en la misma proporción que al resto de la población. Nuevas fuentes de datos relativos a la ocurrencia de óbitos en los domicilios, de alcance nacional, como es el caso del Censo de 2010, pueden contribuir a una mejor comprensión de las desigualdades según color de piel o raza en Brasil.

Indios Sudamericanos; Salud de Poblaciones Indígenas; Mortalidad Infantil; Desigualdades en la Salud
Recebido em 21/Mar/2016

Versão final reapresentada em 18/Set/2016

Aprovado em 22/Set/2016 